Elsevier Editorial system(tm) for Colloids and Surfaces A: Physicochemical and Engineering Aspects

Manuscript Draft

Manuscript Number:

Title: Equilibrium of droplets on a deformable substrate: influence of disjoining pressure

Article Type: SI: Victor_Starov

Keywords: equilibrium droplets, deformable substrate, disjoining pressure

Corresponding Author: Prof. Victor Starov, MSc, PhD, DSC

Corresponding Author's Institution: Loughborough University

First Author: Gulraiz Ahmed

Order of Authors: Gulraiz Ahmed; Victor Starov, MSc, PhD, DSc; Vasily Kalinin

Abstract: Equilibrium of liquid droplets on soft deformable substrates is investigated.. Disjoining pressure action in the vicinity of the apparent three phase contact line is taken into account. It is shown that the disjoining pressure action determines the substrate deformation. A simplified linear disjoining pressure isotherm and simple Winkler's model to account for the substrate deformation are used which allows to deduce an analytical solutions for both the liquid profile and substrate deformation. The apparent equilibrium contact angle that the liquid makes with the substrate is calculated and it's dependency on the system parameters is investigated. 
To whom it may concern

Direct Line:+44 (0)1509 222508

Fax: +44 (0)1509 223923

E-mail: V.M.Starov@Lboro.ac.uk

http://www.lboro.ac.uk/departments/cg/staff/cgvms.htm

$30^{\text {th }}$ March, 2016

Dear Madam/Sir,

We submit of our manuscript Equilibrium of droplets on a deformable substrate:

influence of disjoining pressure by Gulraiz Ahmed, Victor M. Starov, Vasily V.

Kalinin for a publication in a Special Issue "Victor Starov".

Regards, Victor Starov

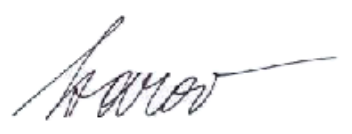

Prof V. M. Starov

Professor Victor M Starov, PhD, DSc,

Fellow of the Royal Society of Chemistry,

Honorary Professor, Moscow State University of Food Production,

Department of Chemical Engineering

Loughborough University

Ashby Rd, Loughborough,

Leicestershire, LE 11 3TU, UK 
Graphical abstract

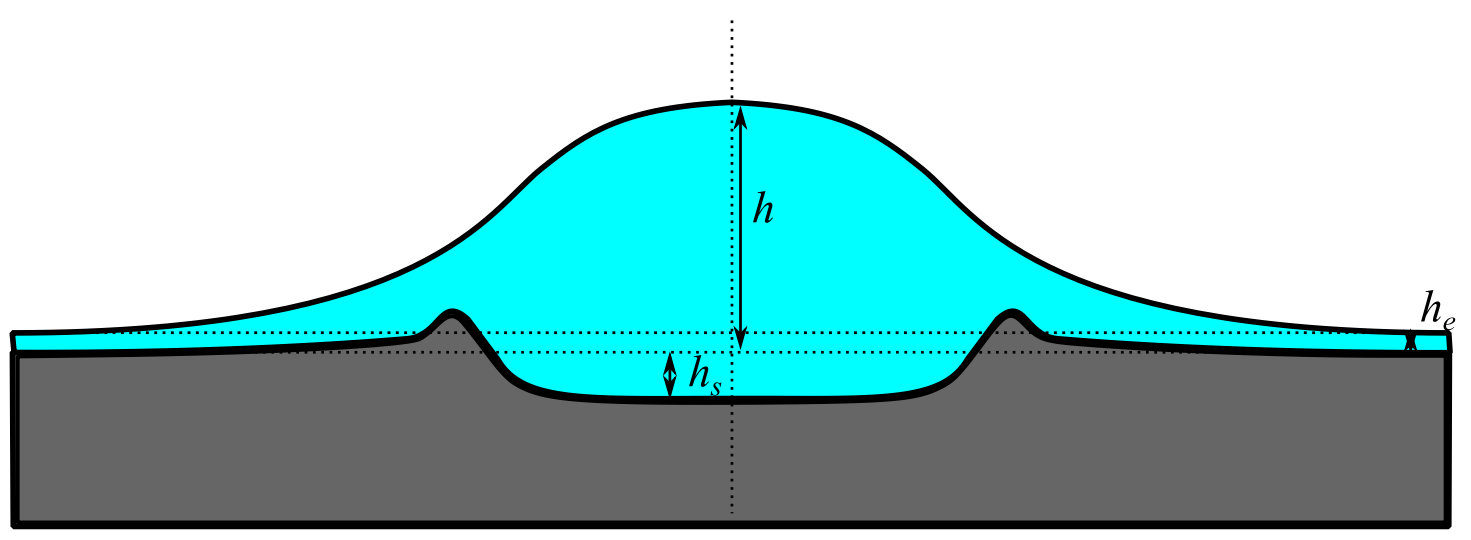


${ }^{*}$ Highlights (for review)

Transition zone

$x^{2}+x^{2}$

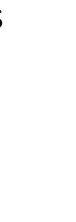

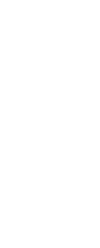

$x^{2}$

(1) 


\title{
Equilibrium of droplets on a deformable substrate: influence of disjoining pressure
}

Gulraiz Ahmed $^{\mathrm{a} *}$, Victor M. Starov ${ }^{\text {a }}$, Vasily V. Kalinin. Gubkin Russian State University of Oil and GasV.V. Kalinin ${ }^{\text {b }}$

${ }^{a}$ Department of Chemical Engineering, Loughborough University, Loughborough, LE11 3TU, UK.

${ }^{\mathrm{b}}$ Gubkin Russian State University of Oil and Gas, Moscow, Russia

* Corresponding author

Email addresses: Gulraiz Ahmed g.ahmed3@1boro.ac.uk.

\begin{abstract}
Equilibrium of liquid droplets on soft deformable substrates is investigated.. Disjoining pressure action in the vicinity of the apparent three phase contact line is taken into account. It is shown that the disjoining pressure action determines the substrate deformation. A simplified linear disjoining pressure isotherm and simple Winkler's model to account for the substrate deformation are used which allows to deduce an analytical solutions for both the liquid profile and substrate deformation. The apparent equilibrium contact angle that the liquid makes with the substrate is calculated and it's dependency on the system parameters is investigated.
\end{abstract}

Key words: equilibrium droplets, deformable substrate, disjoining pressure.

\section{Introduction}

Equilibrium of a liquid droplet on a solid substrate is frequently described based on Young's equation [1]. This simplified equation involves the balance of the horizontal forces leaving the vertical force unbalanced. The latter is possible in the case of a rigid substrate but should be reconsidered in the case of soft deformable substrates. It has been shown in [2] that disjoining pressure action in the vicinity of the apparent three phase contact line results in a deformation of a soft solid substrate.

Note, that in the case of direct application of Young's equation, at the three phase contact line there exists deformation singularity, i.e. the substrate deformation goes to infinity [3-7]. Their investigation revealed that all the equilibrium properties (i.e. contact angle, droplet radius, droplet volume, etc.) of the system under consideration rely upon the selected artificial length parameter which determines a width of zone near the contact line where surface tension is applied. However, 
disjoining pressure is a real physical phenomenon, which determines the substrate deformation of soft surfaces.

The shape of the disjoining pressure isotherm determines the shape and the contact angle of the droplet at equilibrium [2].

The problem of equilibrium of the droplet on a deformable substrate has recently gained a lot of interest. There have been a number of experimental studies recently that have considered liquids on deformable substrates [8-14], where deformation of soft solids was investigated near the apparent contact line, but there exists a gap in theoretically understanding of the problem. This void is primarily due to the stress singularity present at the three phase contact line.

Below a mathematical model is presented which incorporates the effect of both capillary and disjoining pressure isotherm on the substrate deformation.

\section{Disjoining pressure and deformation of soft solids}

In the case of partial wetting, that is, equilibrium contact angle, $\theta_{e}>0$, the shape of the transition zone between the bulk of the liquid and the thin film on substrate can be expressed via the disjoining pressure isotherm, $\Pi(h)$ [2]. The equilibrium excess pressure, $P_{e}$, inside the droplet can be expresses according to Kelvin's equation [2] and the equilibrium contact angle of a two dimensional droplet can be expressed using the disjoining pressure isotherm as follows [2]:

$$
\cos \theta_{e, 2 D}=1+\frac{\Pi\left(h_{e}\right) h_{e}}{\gamma}+\frac{1}{\gamma} \int_{h_{e}}^{\infty} \Pi(h) d h
$$

Eq. (1) corresponds to an S-shaped disjoining pressure isotherm, $\Pi(h)$. Below the transition zone between the flat meniscus and a film having $\theta_{e}>0$ is examined below as an example, following [15]. The liquid droplet forms a wedge changing to flat equilibrium film of thickness, $h_{e}$, far from the droplet (Fig. 1). 


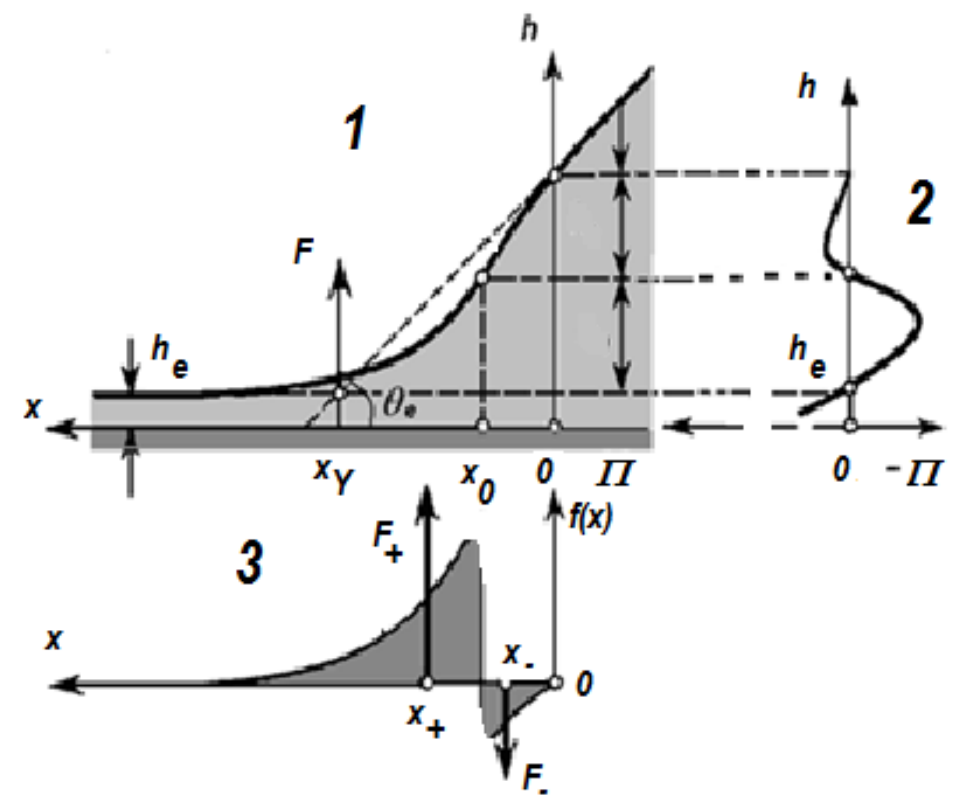

Fig. 1. 1- Profile of transition zone $h(x)$ between bulk liquid and flat wetting film, 2- s shaped disjoining pressure isotherm, $\Pi(h)$, and 3 - profile of normal pressures acting on substrate; $x_{Y}$ is the position where the vertical force is exerted.

The origin is taken at $x=0$, which is a point on the profile lying beyond the influence of surface forces (Fig. 1). The profile of the transition zone, $h(x)$, can be calculated according to Eq. (2), which includes the influence of capillary and disjoining pressure isotherm [2]:

$$
\gamma K(x)+\Pi(h)=\frac{\gamma h^{\prime \prime}}{\left[1+\left(h^{\prime}\right)^{2}\right]^{3 / 2}}+\Pi(h)=P_{e},
$$

where, $h^{\prime}=d h / d x ; h^{\prime \prime}=d^{2} h / d x^{2}$; and $P_{e}$ is the excess pressure in the droplet. In the region of the flat equilibrium film, $h^{\prime \prime}=0$ and $\Pi\left(h_{e}\right)=P_{e}$. In bulk of the liquid, beyond the influence of the surface forces, $\Pi=0$ and $P_{e}=\gamma / R$, where $R$ is the radius of curvature of the droplet. In case of planar wedge (Fig. $1, R=\infty$ and $P_{e}=0$. Therefore from Eq. (2) can be concluded:

$$
\Pi(h)=-\frac{\gamma h^{\prime \prime}}{\left[1+\left(h^{\prime}\right)^{2}\right]^{3 / 2}} .
$$

The resultant forces on the substrate is given by the following equation:

$$
F=\int_{0}^{\infty} \Pi(h) d x
$$

Substituting the disjoining pressure isotherm $\Pi(h)$ according to Eq. (3) in equation (4) results in:

$$
\begin{gathered}
F=-\gamma \int_{0}^{\infty}\left\{\frac{h^{\prime \prime}}{\left[1+\left(h^{\prime}\right)^{2}\right]^{\frac{3}{2}}}\right\} d x=\gamma \frac{h^{\prime}(0)}{\sqrt{\left[1+\left(h^{\prime}(0)\right)^{2}\right]}}=\frac{\gamma \tan \theta_{e}}{\sqrt{\left[1+\tan ^{2} \theta_{e}\right]}} \\
=\gamma \sin \theta_{e}
\end{gathered}
$$


Boundary conditions used in the expression above (equation (5)) are, $h^{\prime}(\infty)=0$ and $h^{\prime}(0)=$ $\tan \theta_{e}$. This shows that the integration performed over the local values leads to same expression as the vertical component of the surface tension from the Young's equation. In contrast to Young's, the force is not exerted on a specific point, but is distributed over the region where disjoining pressure acts, i.e. transition zone. Based on this conclusion a mathematical model is derived in the next section.

\section{Mathematical model}

In this section a mathematical model is derived for a liquid droplet on a deformable substrate. According to Winkler's model there is a linear relationship between the local deformation and the applied local stress $[16,17]$. Winkler's model is used below.

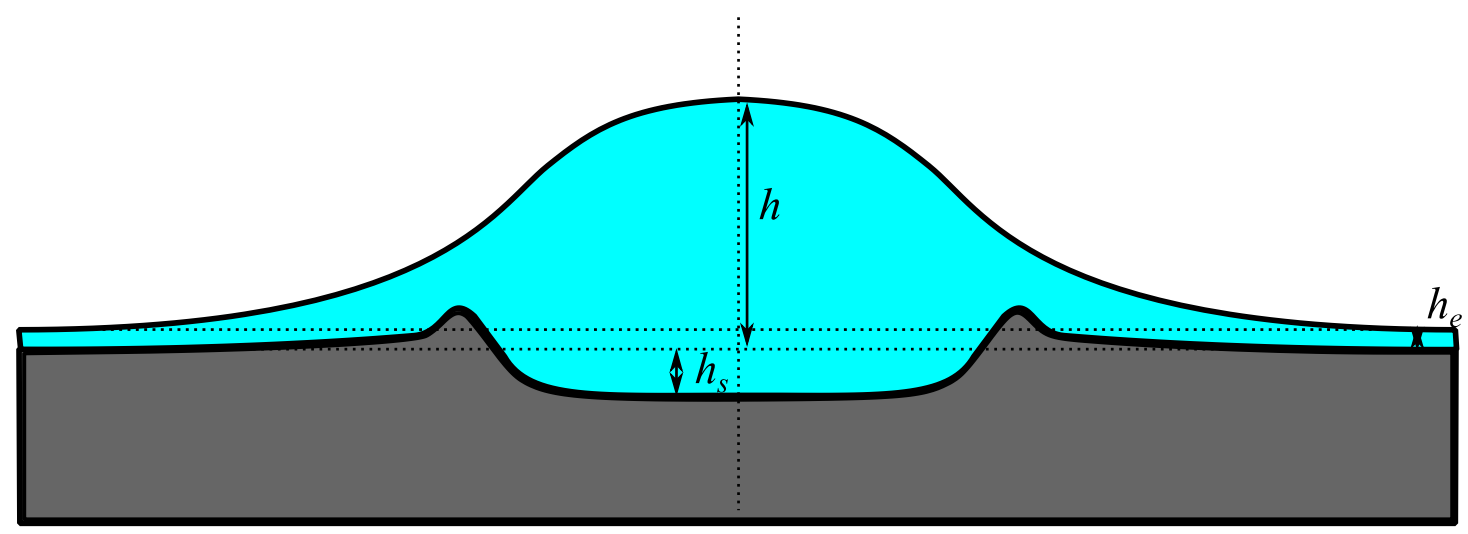

Fig. 2. Schematic diagram of the liquid droplet on a deformable substrate.

Deformation in the soft substrate is local and is directly proportional to the applied pressure, $P$. According to Winkler's model,

$$
h_{s}=-K P \text {, }
$$

where $K$ is the elasticity coefficient, $h_{s}$ is the local deformation of the substrate due to the presence of the droplet above.

The pressure in the ambient air is $P_{\text {air }}$. Under the action of the pressure from the ambient air the solid deformation is:

$$
h_{s e}=-K P_{\text {air }}
$$

The deformed solid substrate is covered by equilibrium liquid thin film, which is calculated according to well-known Kelvin's equation: 


$$
\Pi\left(h_{e}\right)=P_{e}=\frac{R T}{v_{m}} \ln \frac{p_{\text {sat }}}{p}
$$

where, $v_{m}$ is the molar volume of the liquid, $T$ is the temperature, $R$ is the gas constant; vapour pressure, $p$, which is higher than he saturated pressure $p_{\text {sat }}$. Reminder, a droplet can be at the equilibrium with oversaturated vapour pressure.

The excess free energy of the equilibrium thin film of deformed solid per unit area is given by,

$$
\frac{F_{e, f i l m}}{S_{\text {film }}}=\gamma+\gamma_{s}+P_{e} h_{e}+\frac{h_{s e}^{2}}{2 K}+\int_{h_{e}}^{\infty} \Pi(h) d h,
$$

where, $P_{e}=P_{\text {air }}-P_{\text {liquid }}, \gamma$ and $\gamma_{\mathrm{s}}$ are liquid-vapour and solid-vapour interfacial tensions. This free energy should be subtracted from the free energy of the droplet, otherwise the excess free energy of the droplet will be infinite. The excess free energy of the droplet is as follows,

$$
F-F_{e, \text { film }}=\gamma \Delta S+\gamma_{s} \Delta S_{S}+\Delta V+F_{\text {surface forces }}+F_{\text {deformation }}
$$

where $\Delta$ means "as compared with a flat equilibrium film". Therefore Eq. (10) can be rewritten as:

$$
F-F_{e, f i l m}=2 \pi \int_{0}^{\infty} r f\left(h, h^{\prime}, h_{s}, h_{s}^{\prime}\right) d r
$$

where

$$
f\left(h, h^{\prime}, h_{s}, h_{s}^{\prime}\right)=r\left\{\begin{array}{c}
\gamma \sqrt{1+h^{\prime 2}(r)}-\gamma+\gamma_{s} \sqrt{1+h^{\prime 2}(r)}-\gamma_{s}+ \\
P_{e}\left(h-h_{s}\right)-P_{e} h_{e}+ \\
{\left[\frac{h_{s}^{2}}{2 K}-\frac{h_{s e}^{2}}{2 K}\right]+\int_{h-h_{s}}^{\infty} \Pi(h) d h-\int_{h_{e}}^{\infty} \Pi(h) d h}
\end{array}\right\}
$$

The expression under the integral in Eq. (11) tends to zero as $r$ tends to infinity.

Under equilibrium conditions the excess free energy should reach to a minimum value. To satisfy this condition the first variation of free excess energy should be zero, which results in two Euler equations for the droplet and soft substrate profiles,

$$
\begin{gathered}
\frac{d}{d r}\left(\frac{\partial f}{\partial h^{\prime}}\right)-\frac{\partial f}{\partial h}=0 \\
\frac{d}{d r}\left(\frac{\partial f}{\partial h_{s}^{\prime}}\right)-\frac{\partial f}{\partial h_{s}}=0
\end{gathered}
$$

Substitution the expression for $f$ from (12) into equations (13) and (14) results in the following equations:

$$
\frac{\gamma}{r} \frac{d}{d r} \frac{r h^{\prime}}{\left(1+h^{\prime 2}\right)^{1 / 2}}+\Pi\left(h-h_{s}\right)=P_{e}
$$




$$
\frac{\gamma_{s}}{r} \frac{d}{d r} \frac{r h_{s}^{\prime}}{\left(1+h_{s}^{\prime 2}\right)^{1 / 2}}-\Pi\left(h-h_{s}\right)-\frac{h_{s}}{K}=-P_{e}
$$

These two equations (15) and (16) forms a system of two differential equations for two unknown profiles: the liquid droplet, $h(r)$, and deformed solid substrates, $h_{s}(r)$.

Below the simplest case is considered, $\gamma_{s}=0$. Eq.(16) in this case results in:

$$
h_{s}=-K\left[\Pi\left(h-h_{s}\right)-P_{e}\right]
$$

Region where surface forces are negligible, i.e. bulk of the droplet, equation (17) gives:

$$
h_{s}=K P_{e}<0
$$

because $P_{e}$ is negative. Hence, Eq. (17) is in agreement with definition (6). In the case of a low slope approximation, $h^{\prime 2} \ll 1$ the profile of the droplet $h(r)$ and profile of the substrate $h_{s}(r)$ satisfy the following set of a second order ordinary differential equation and an an algebraic equation:

$$
\begin{gathered}
\gamma\left(h^{\prime \prime}+\frac{h^{\prime}}{r}\right)+\Pi\left(h-h_{s}\right)=P_{e} \\
h_{s}=-K\left[\Pi\left(h-h_{s}\right)-P_{e}\right]
\end{gathered}
$$

Eq. (19) is different from the usual capillary equation for the droplet on a rigid substrate, because now the disjoining pressure term depends on the profile of the deformable substrate, $h_{s}(r)$, which is determined according to Eq. (20). Both Eq. (19) and (20) are coupled and can be solved numerically only, however, below the problem is simplified even further to obtain an analytical solution. For this purpose we adopt a very simple disjoining pressure isotherm (linear function of $h$ (Fig. 3)):

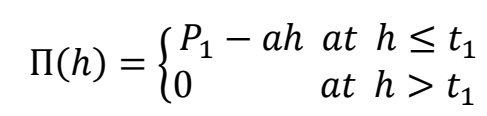

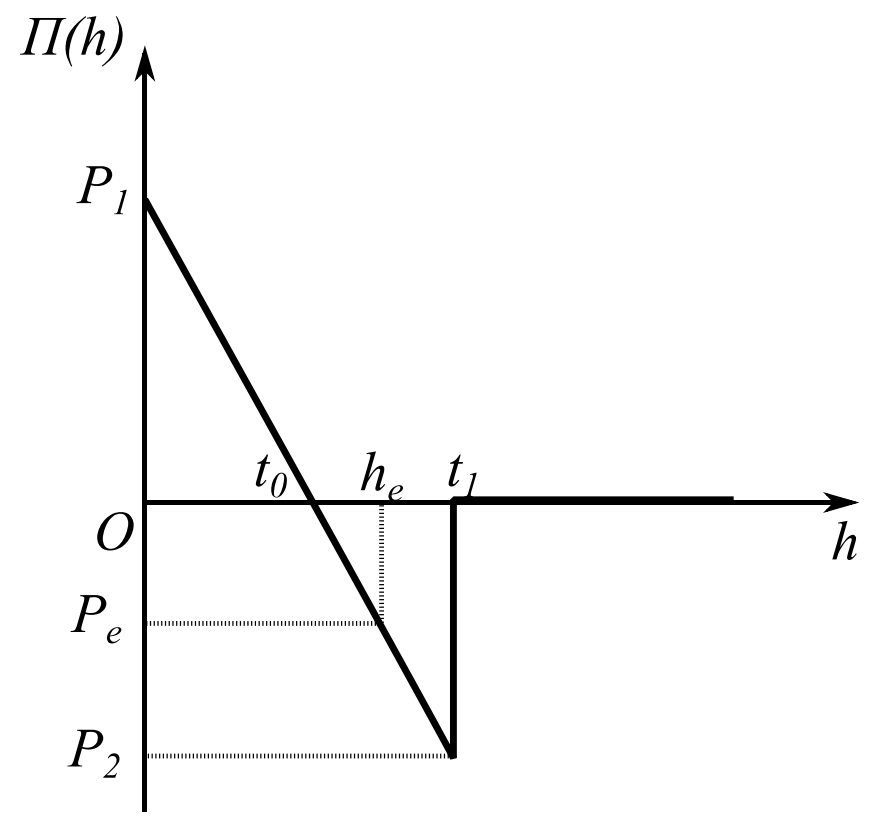


Fig.3. Simplified disjoining pressure isotherm adopted below for calculations.

where $P_{1}$ and $t_{0}$ are defined in Fig. 3, $t_{1}$ is the range of surface forces action. The slop $a$ of the $\Pi(h)$ dependency is given by:

$$
a=\frac{P_{1}-P_{e}}{h_{e}}
$$

The selected linear dependency of the disjoining pressure isotherm $\Pi(h)$ on $h$ according to Eq. (21) in spite of considerable simplification still captures the essential properties of the disjoining pressure isotherm (i) it satisfies the stability condition, $\Pi^{\prime}(h)<0$ when $h<t_{1}$, and (ii) the influence of surface forces is short range and radius of it's action is defined by $t_{1}$; (iii) at the proper choice of the disjoining pressure parameters (see below) it corresponds to the particle wetting case.

\subsection{Droplets on a non-deformable substrate}

Fig. 4 shows a schematic diagram of the droplet on the non-deformable solid substrate, where $L_{1}$ is the length from the origin to the point where the influence of the surface forces come into play, $L$ is the effective radius of the droplet base, $\theta_{e, n d}$ is the equilibrium contact angle the droplet makes with the solid substrate, $R$ is the radius of the droplet.

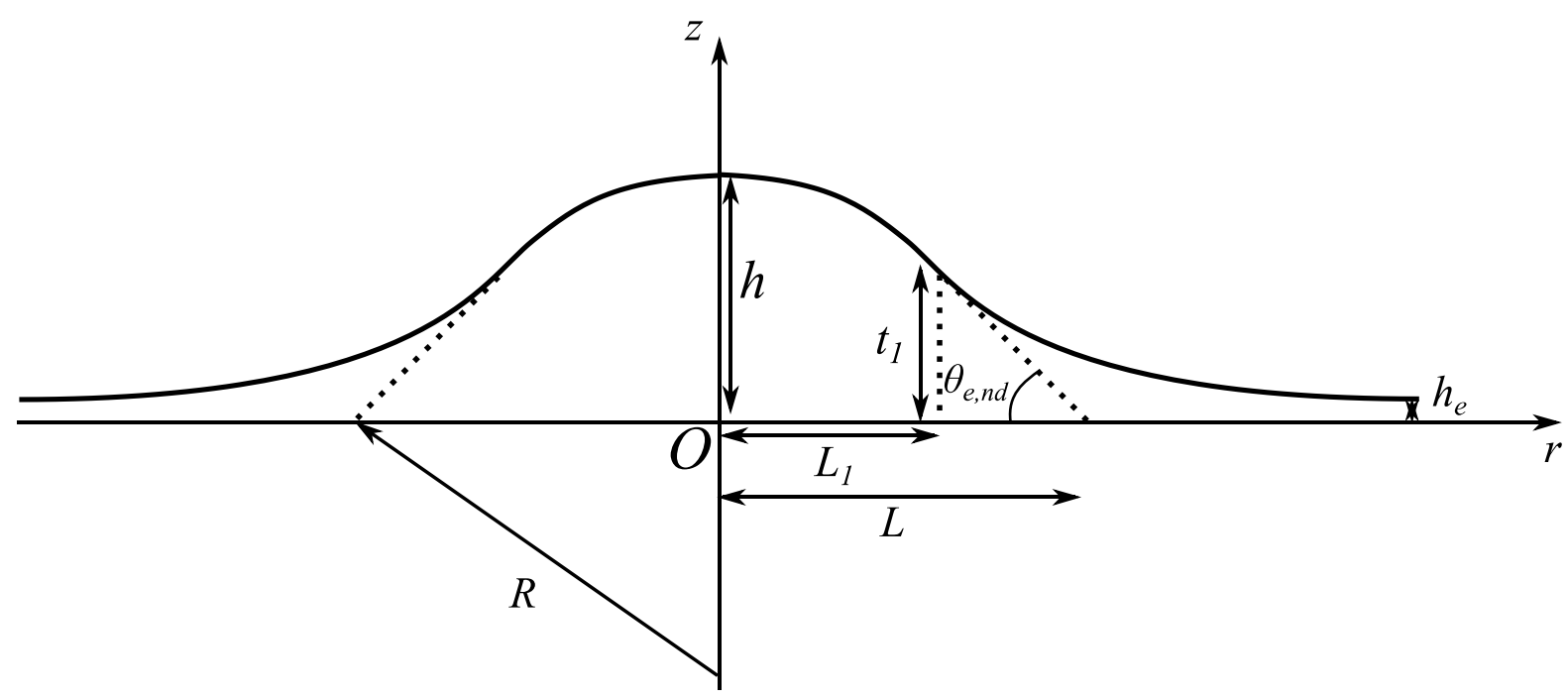

Fig. 4. Schematic diagram of a droplet on a non-deformable solid substrate. Here, $R=-\frac{\gamma}{P_{e}}$.

For a solid non-deformable substrate Eq. (19) reduces to:

$$
\gamma\left(h^{\prime \prime}+\frac{h^{\prime}}{r}\right)+\Pi(h)=P_{e}
$$


For the bulk of the liquid droplet, i.e. the spherical region, at $h>t_{1}$ and $\Pi(h)=0$. Hence, in this region Eq.0 (23) transforms into:

$$
\gamma\left(h^{\prime \prime}+\frac{h^{\prime}}{r}\right)=P_{e}
$$

Solution of this equation is:

$$
h(r)=A r^{2}+B,
$$

where integration constants, $A$ and $B$, can be determined from boundary conditions at $\mathrm{r}=0$ and $\mathrm{r}=\mathrm{L}$ and this solutions is marked by a subscript out: out

$$
h_{\text {out }}(r)=-\frac{r^{2}}{2 R}+\frac{R \sin ^{2} \theta_{e, n d}}{2} \text { or } h_{\text {out }}(r)=\frac{-r^{2}+L^{2}}{2 R}
$$

For the transition region in the case of non-deformable solid substrate Eq. (23) along with the disjoining pressure isotherm given by Eq. (21) results in:

$$
h^{\prime \prime}+\frac{h^{\prime}}{r}-\frac{a h}{\gamma}=\frac{P_{e}-P_{1}}{\gamma}
$$

Solution of Eq. Error! Reference source not found. is as follows:

$$
h_{i n}=h_{e}+C_{1, n d} K_{0}(r \lambda)
$$

where, $\lambda=\sqrt{\frac{a}{\gamma}}, K_{0}$ is the modified Bessel function of the zero order, $C_{1, n d}$ is an integration constant and this solution is marked by the subscript in. Solutions (25) and (28) should satisfy the following boundary conditions:

$h_{\text {out }}\left(L_{1}\right)=h_{\text {in }}\left(L_{1}\right)=t_{1} ; h_{\text {out }}^{\prime}\left(L_{1}\right)=h_{\text {in }}^{\prime}\left(L_{1}\right)$, which gives three equations for determination of the three unknown coefficients: $C_{1, n d}, \theta_{e, n d}$ and $L_{l}$.

Solution results in

$$
\begin{aligned}
& C_{1, n d} K_{0}\left(L_{1} \lambda\right)=\frac{L_{1}}{R \lambda}, \\
& \theta_{e, n d}=\sqrt{\frac{2}{R}\left(h_{e}+\frac{L_{1}}{R \lambda}+\frac{L_{1}^{2}}{2 R}\right),} \\
& L_{1}=R \lambda\left(t_{1}-h_{e}\right) .
\end{aligned}
$$

The latter allows determining the effective radius of the droplet, $\mathrm{L}$ :

$$
L=\sqrt{L_{1}^{2}+2 R t_{1}} .
$$

Introducing: $\quad P_{2}=a\left(t_{0}-t_{1}\right)$, Eq. (30) can be rewritten as

$$
\theta_{e, n d}=\sqrt{\frac{1}{\gamma a}\left[P_{e}^{2}-P_{1} P_{e}-P_{2} P_{e}+P_{2}^{2}\right]}
$$


The equilibrium contact angle was previously determined according to Eq. (1). According to the simplifies isotherm (Fig. 3) this contact angle can be calculated as:

$$
\theta_{e, 2 D}=\sqrt{2\left\{-\frac{P_{e} h_{e}}{\gamma}-\frac{a}{\gamma}\left[t_{0}\left(t_{1}-h_{e}\right)-\frac{1}{2}\left(t_{1}^{2}-h_{e}^{2}\right)\right]\right\}}
$$

\subsection{Deformable substrate}

Fig. 5 shows a schematic diagram of a droplet on the deformable solid substrate based on the assumption of a linear disjoining pressure, according to Eq. (21) (Fig.3). In Fig. $5 \theta_{e, d}$ is the equilibrium macroscopic contact angle of the droplet with the soft substrate.

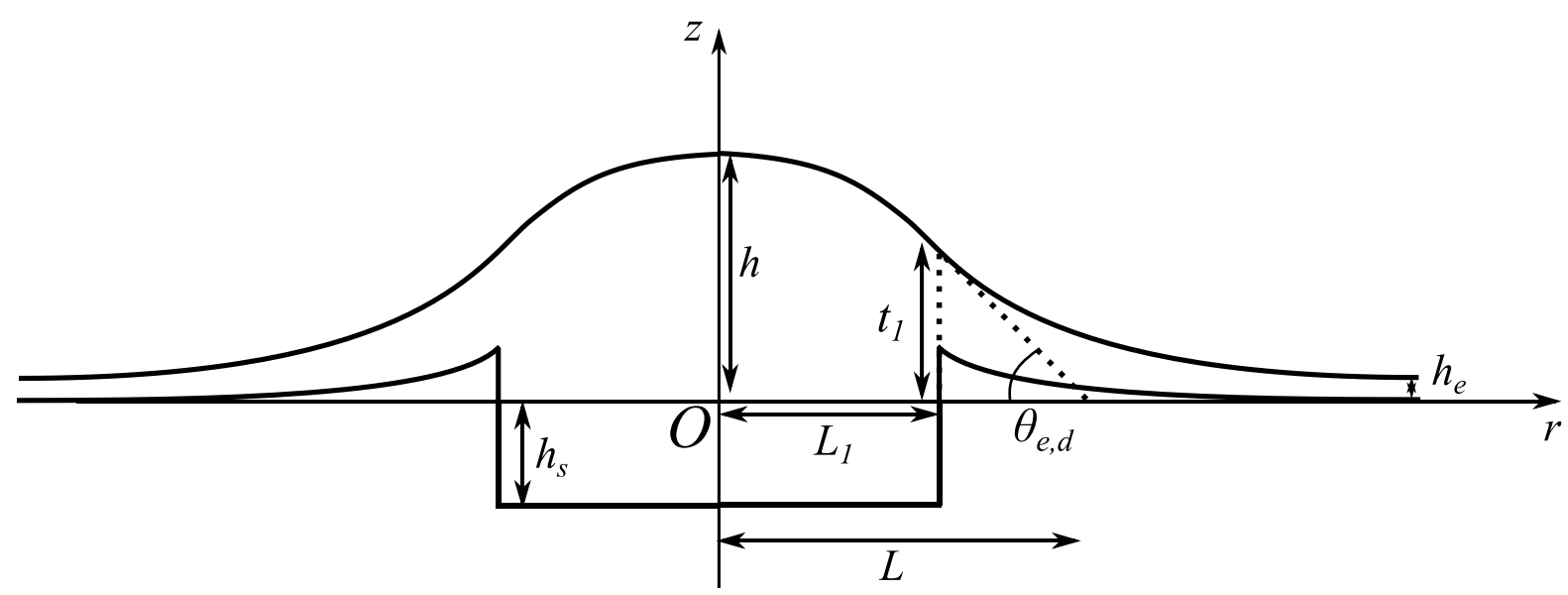

\section{Fig. 5. Schematic diagram of droplet on a deformable/soft substrate}

For the bulk of the liquid droplet, i.e. the spherical region, $h-h_{s}>t_{1}$, that is at $0<\mathrm{r}<\mathrm{L}_{1}$ (Fig. 5) from Eq. (21):

$$
h_{s}=K P_{e}
$$

For the transition region, that is at $r>L_{1}$ Eq. (20)gives:

$$
h_{s}=\frac{K\left(P_{e}-P_{1}+a h\right)}{a K+1}
$$

Substitution of the latter expression into Eq. (20) results in:

$$
h^{\prime \prime}+\frac{h^{\prime}}{r}-\frac{a}{\gamma(a K+1)} h=\frac{P_{e}-P_{1}}{\gamma(a K+1)}
$$

Solution of the latter equation is: $h=h_{e}+C_{1, d} K_{0}(r \beta)$,

where $\beta=\sqrt{\frac{a}{\gamma(a K+1)}}$ and $C_{l, d}$ is an integration constant. 
The droplet profile and its derivative should be continuous at $\mathrm{r}=\mathrm{L}_{1}$, but the solid surface should be discontinuous and $\mathrm{h}\left(\mathrm{L}_{1}\right)-\mathrm{h}_{\mathrm{s}}\left(\mathrm{L}_{1}\right)=\mathrm{t}_{1}$. Using these boundary conditions all unknown constants $C_{l, d}, \theta_{e, d}$ and $L$, can be determined. Below only expression for the contact angle on the deformable substrate is presented:

$$
\theta_{e, d}^{2}=\frac{K P_{2}}{\gamma}\left(P_{2}-P_{e}\right)+\theta_{e, n d}^{2}
$$

where $P_{2}=a\left(t_{0}-t_{1}\right)$. That is if $K=0$ (non-deformable substrate), then $\theta_{e, d}=\theta_{e, n d}$. Expression $\frac{K P_{2}}{\gamma}\left(P_{2}-P_{e}\right)$ in Eq. (37) is always positive, that is, $\theta_{e, d}>\theta_{e, n d}$ the contact angle on a deformable substrate is always bigger than the contact a angle on the corresponding non-deformable substrate. 


\section{Results and Discussion}

\subsection{Non-deformable substrate: Effect of variation of $P_{e}$ on $\theta_{e, n d}$}

Dependency of equilibrium contact angles $\theta_{e, n d}$ and $\theta_{e, 2 D}$ from according to Eqs. (280) and (34) on $P_{e}$ are presented in this section. Below $\left|P_{e}\right|$ is varied from 0 to $\left|P_{2}\right|$. Fig. 6 shows the behaviour of equilibrium contact angle when the excess pressure is varied. Fig. 6a shows the variation at $t_{0}=7 \times 10^{-7} \mathrm{~cm}$ and Fig. $6 \mathrm{~b}$ shows variation at $t_{0}=2 \times 10^{-6} \mathrm{~cm}$. In both cases, two-dimensional equilibrium contact angle $\theta_{e, 2 D}$ calculated according to Eq (34) turns up to be higher as compared with three-dimensional equilibrium contact angle calculated according to Eq. (33). It was shown earlier [2] $\theta_{e, 2 D}$ can only increase with the decrease in $\mathrm{P}_{\mathrm{e}}$, which is in agreement with both Figs. 6a and $6 \mathrm{~b}$. However, it turns out that the dependency of the contact angle in the three dimensional case, $\theta_{e, n d}$, differs substantially from the two dimensional case (always higher) and can go via minimum as a function of $\mathrm{P}_{\mathrm{e}}$ (Fig. 6a). If the value of $t_{0}$ is increased and brought closer to $t_{1}$ then $\theta_{e, n d}$ is an increasing function of $P_{e}$ with no minimum.

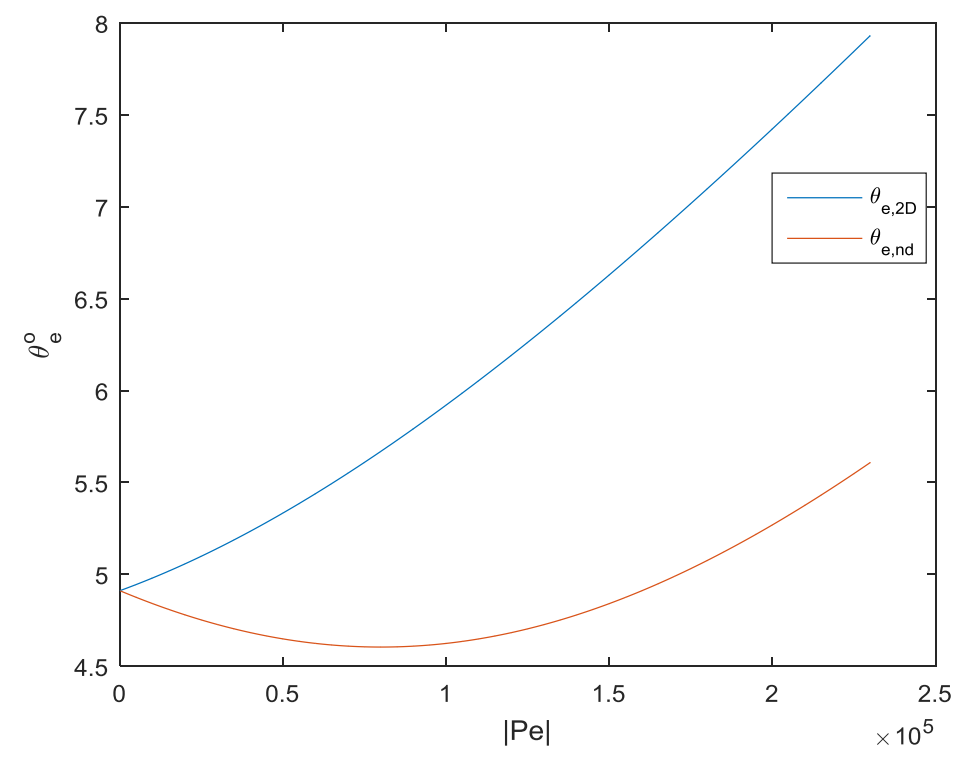

(a) $t_{0}=7 \times 10^{-7} \mathrm{~cm}$ 


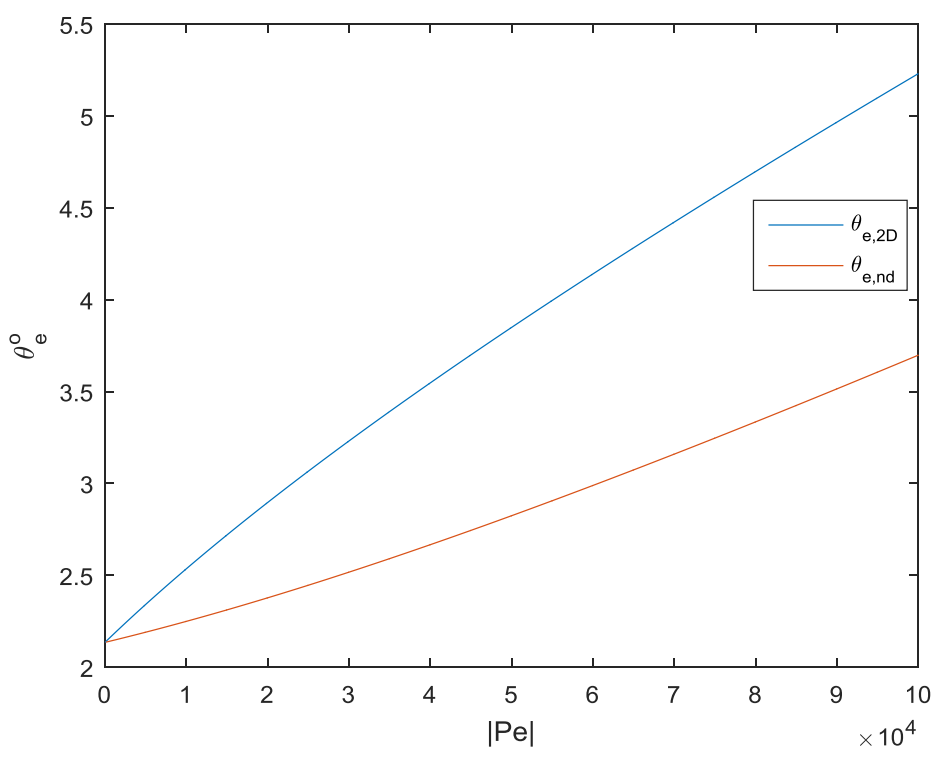

(b) $t_{0}=2 \times 10^{-6} \mathrm{~cm}$

Fig. 6. No-deformable substrate. Effect of variation of excess pressure, $P_{e}$ on two-dimensional, $\boldsymbol{\theta}_{e, 2 D}$, and three-dimensional, $\boldsymbol{\theta}_{e, n d}$, equilibrium contact angles. Selected parameters are as shown

\begin{tabular}{|l|l|}
\hline Physical Property & Value \\
\hline Surface tension, $\gamma$ & $72 \mathrm{dyn} / \mathrm{cm}$ \\
\hline$t_{1}$ & $3 \times 10^{-6} \mathrm{~cm}$ \\
\hline$a$ & $1 \times 10^{11} \mathrm{dyn} / \mathrm{cm}^{3}$ \\
\hline$\left|P_{e}\right|$ & $0-\left|P_{2}\right| \mathrm{dyn} / \mathrm{cm}^{2}$ \\
\hline
\end{tabular}

\subsection{Deformable substrate}

The profile of the droplet and the subsequent changes in the deformable substrate are plotted in Fig. using equations deduced in section 3.2. The soft substrate behaves in the same manner as predicted in Fig. 5. 


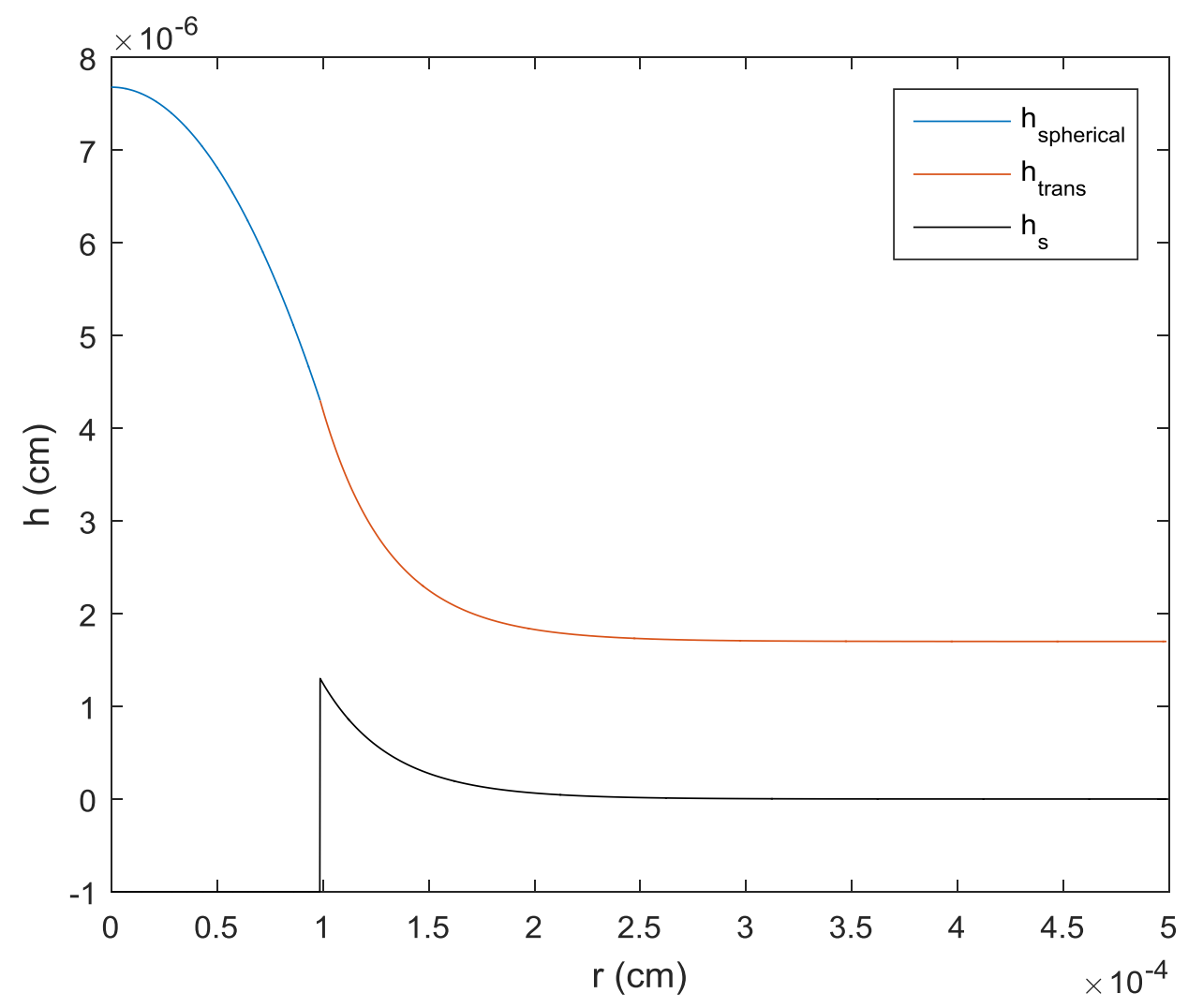

Fig. 6. Deformable substrate. Calculated profile of the droplet and deformable substrate.

\begin{tabular}{|l|l|}
\hline Physical Property & Value \\
\hline Surface tension, $\gamma$ & $72 \mathrm{dyn} / \mathrm{cm}$ \\
\hline$t_{1}$ & $3 \times 10^{-6} \mathrm{~cm}$ \\
\hline$t_{0}$ & $7 \times 10^{-7} \mathrm{~cm}$ \\
\hline$a$ & $1 \times 10^{11} \mathrm{dyn} / \mathrm{cm}^{3}$ \\
\hline$K$ & $1 \times 10^{-11} \mathrm{~cm}^{3} / \mathrm{dyn}$ \\
\hline
\end{tabular}

\subsection{Effect of variation of $P_{e}$ on equilibrium contact angle $\theta_{e, d}$}

Excess pressure is varied as before $0 \leq\left|P_{e}\right| \leq\left|P_{2}\right|$. In order to compare the results with the $\theta_{e, n d}$, the value of $K$ is increased gradually from 0 to $1 \times 10^{-11}$. All other physical properties of the disjoining pressure isotherm are kept the same as shown in Fig. 6 . for $t_{0}$ which is equal to $7 \times$ $10^{-7} \mathrm{~cm}$ in Fig. 7a and is equal to $2 \times 10^{-6} \mathrm{~cm}$ in Fig. 7b. Fig. 7a has a minima for $\theta_{e, d}$ and it becomes an increasing function as $t_{0}$ is increased and brought closer to $t_{1}$ (Fig. 7b). It is apparent from Fig. 7 that $\theta_{e, d}$ is bigger than $\theta_{e, n d}$ for all values of $K>0$. 


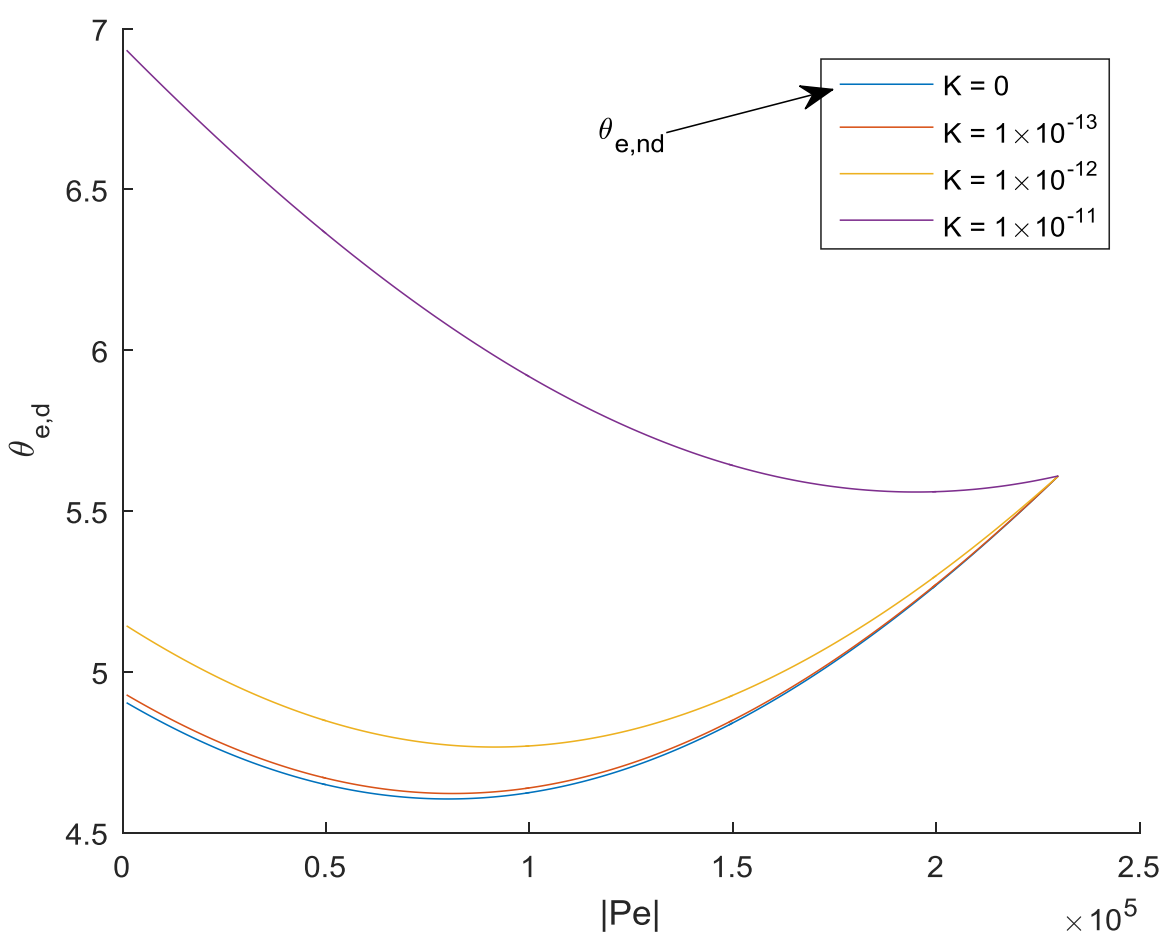

(a) $t_{0}=7 \times 10^{-7} \mathrm{~cm}$

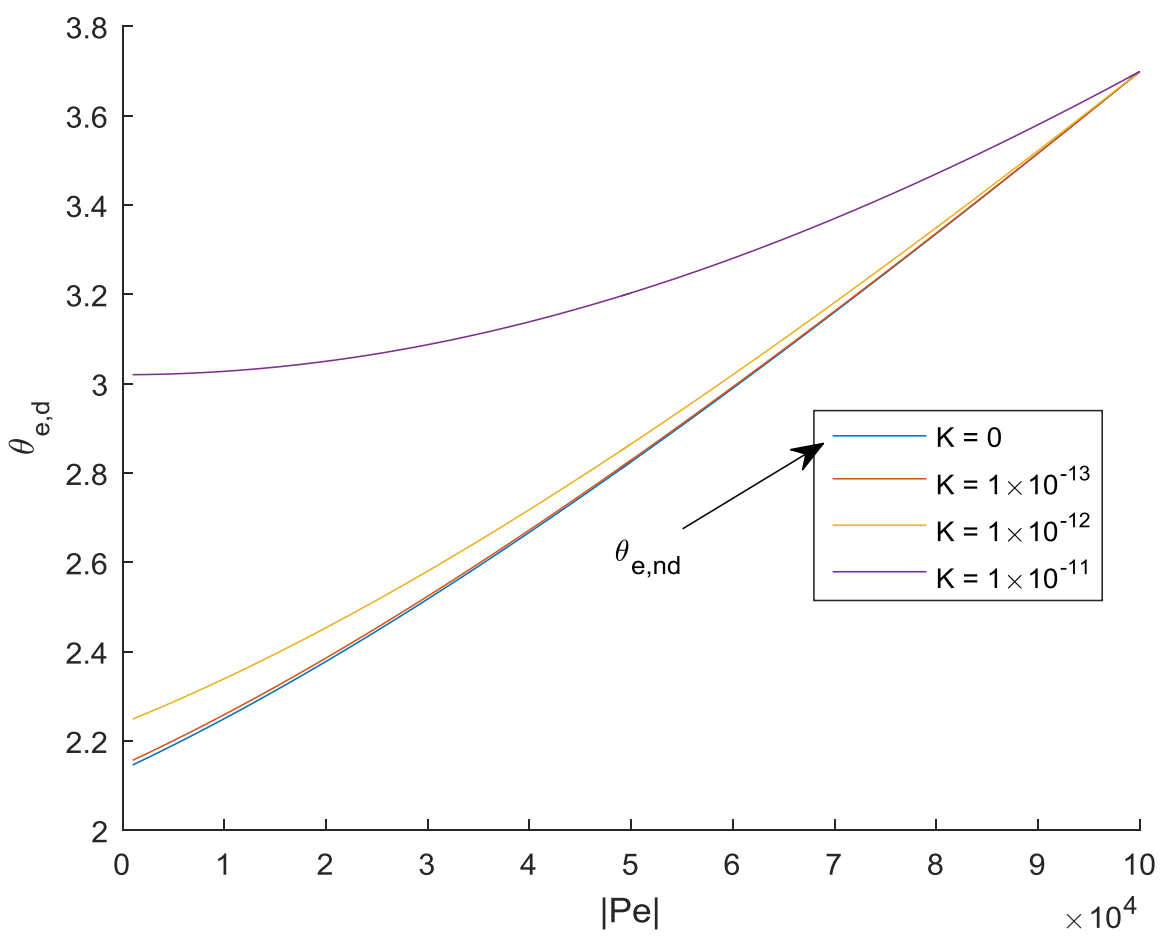

(a) $t_{0}=2 \times 10^{-6} \mathrm{~cm}$

Fig. 7. Effect of variation of excess pressure, $P_{e}$ on $\theta_{e, d}$ for different values of elasticity coefficient $K$. 


\section{Conclusions}

Equilibrium of liquid droplets on soft deformable substrates is investigated. Disjoining pressure action in the vicinity of the apparent three phase contact line is taken into account. It is shown that the disjoining pressure action determines the substrate deformation. A simplified linear disjoining pressure isotherm and simple Winkler's model to account for the substrate deformation are used which allows deducing an analytical solutions for both the liquid profile and substrate deformation. The apparent equilibrium contact angle that the liquid makes with the substrate is calculated and its dependency on the system parameters is investigated. It is shown also that the calculated equilibrium contact angles in the case of non-deformable substrates in the case of two-dimentional droplets are bigger as compared with three dimensional case. It is shown that equilibrium contact angles on deformable substrates are always higher than on the corresponding non-deformable substrates.

\section{Acknowledgements}

This research was supported by CoWet Marie Curie EU project; COST action MP1106, EU and MAP EVAPORATION project, European Space Agency.

\section{References}

1. T. Young, An Essay on the Cohesion of Fluids, Philos. Trans. R. Soc. London. 95 (1805) 65-87.

2. V.M. Starov, M.G. Velarde, C.J. Radke, Wetting and Spreading Dynamics, CRC Press Taylor and Francis Group, 2007.

3. A. Rusanov, Theory of wetting of elastically deformed bodies. 1. Deformation with a finite contact-angle., Colloid J. 37 (1975) 614-622.

4. A. Rusanov, Theory of wetting of elastically deformed bodies. 2. Equilibrium conditions and work of deformation with a finite contact angle, Colloid J. 37 (1975) 623-628.

5. A. Rusanov, Thermodynamics of deformable solid-surfaces, J. Colloid Interface Sci. 63 (1978) 330-345.

6. M.E.R. Shanahan, The Spreading Dynamics of a Liquid-Drop on a Viscoelastic Solid, J. Phys. D-Applied Phys. 21 (1988) 981-985.

7. P. Shanahan, MER; Degennes, The ridge produced by a liquid near the triple line solid liquid fluid, Comptes Rendus L Acad. Des Sci. Ser. II. 302 (1986) 517-521.

8. A. Carre, M.E.R. Shanahan, Viscoelastic Braking of a Running Drop, Langmuir. (2001) 2982-2985. 
9. E.R. Jerison, Y. Xu, L.A. Wilen, E.R. Dufresne, Deformation of an Elastic Substrate by a Three-Phase Contact Line, Phys. Rev. Lett. 106 (2011) 186103.

10. S.J. Park, B.M. Weon, J.S. Lee, J. Lee, J. Kim, J.H. Je, Visualization of asymmetric wetting ridges on soft solids with X-ray microscopy., Nat. Commun. 5 (2014) 4369.

11. R.D. Schulman, K. Dalnoki-Veress, Liquid Droplets on a Highly Deformable Membrane, Phys. Rev. Lett. 115 (2015) 206101.

12. M. Shanahan, A. Carre, Viscoelastic dissipation in wetting and adhesion phenomena, Langmuir. (1995) 1396-1402.

13. R.W. Style, R. Boltyanskiy, Y. Che, J.S. Wettlaufer, L.A. Wilen, E.R. Dufresne, Universal Deformation of Soft Substrates Near a Contact Line and the Direct Measurement of Solid Surface Stresses, Phys. Rev. Lett. 110 (2013) 066103.

14. R.W. Style, Y. Che, S.J. Park, B.M. Weon, J.H. Je, C. Hyland, et al., Patterning droplets with durotaxis, Proc. Natl. Acad. Sci. 110 (2013) 12541-12544.

15. B. V. Derjaguin, V.M. Starov, N. V. Churaev, Pressure on a Wetting Perimeter, Kolloidn. Zhurnal. 44 (1982) 871-876.

16. E. Winkler, The theory of elasticity and strength with special reference to their application in the art for polytechnics, building academies, engineers, mechanical engineers, Architects, Etc., in: H. Dominicus, 1867.

17. A.D. Kerr, Elastic and Viscoelastic Foundation Models, J. Appl. Mech. 31 (1964) 491. 


\begin{abstract}
Equilibrium of liquid droplets on soft deformable substrates is investigated.. Disjoining pressure action in the vicinity of the apparent three phase contact line is taken into account. It is shown that the disjoining pressure action determines the substrate deformation. A simplified linear disjoining pressure isotherm and simple Winkler's model to account for the substrate deformation are used which allows to deduce an analytical solutions for both the liquid profile and substrate deformation. The apparent equilibrium contact angle that the liquid makes with the substrate is calculated and it's dependency on the system parameters is investigated.
\end{abstract}

